\title{
Perioperative planning in the COVID-19 pandemic: Cardiovascular perfusion and device-related issues
}

\author{
COVID-19 pandemisinde perioperatif planlama: \\ Kardiyovasküler perfüzyon ve cihaza bağlı sorunlar \\ Serdar Günaydın (1) \\ Department of Cardiovascular Surgery, Ankara City Hospital, Ankara, Turkey
}

The COVID-19 pandemic puts health services, including cardiac surgery units, under escalating pressure. The Heart Team is confronted with a novel virus and disease which leads to a great uncertainty. However, the decision of the operation is inevitable which is deemed time-sensitive, as delaying the procedure may cause harm to the patient. ${ }^{[1]}$ I would like to highlight the vital considerations in the management of extracorporeal circulation and related equipment perspective in the cardiac operation room.

Early reports described up to $29 \%$ infection rates among healthcare professionals. ${ }^{[2]}$ All procedures involving virus infection should be performed by an experienced staff. Patients must be prepared in negative pressure isolation rooms. All laboratory samples should be handled carefully. Procedures should be performed with appropriate personal protective equipment for airborne plus contact precaution including N95/filtering face piece 2 (FFP2) mask, gown, cap, eye protection. Heart-lung machine should be accepted as the principal source of splashing and aerosol generation; therefore, a higher level of protection (e.g., gown at AAMI Level 3 or equivalent) should be considered.

During management of cardiopulmonary bypass (CPB) and anesthesiology, the team must be aware that patients with this infection have significantly deranged levels of coagulation/inflammation parameters: elevated white blood cell count (1.5-fold), neutrophil count (1.7-fold), lower lymphocyte count (0.9-fold), higher
LDH (2.1-fold), alanine aminotransferase (1.5-fold), aspartate aminotransferase (1.8-fold), total bilirubin (1.2-fold), creatinine (1.1-fold), cardiac troponin I (2.2-fold), D-dimer (2.5-fold), and procalcitonin (1.2-fold). Compared to healthy controls, prothrombin time activity was lower and thrombin time shorter. ${ }^{[2]}$ Heparinization from the upper limit is recommended. Activated clotting time is not, then, a potent indicator. Viscoelastic testing is the most optimal option, if available. If not, activated partial thromboplastin time is a more useful test for monitorization. If femoral artery cannulation is a must, novel bidirectional cannula with satisfactory distal limb perfusion rates may be used to avoid ischemia. In patients with multiple thrombosis of circuitry during CPB or extracorporeal membrane oxygenation (ECMO)/extracorporeal life support (ECLS), direct thrombin inhibitors may be alternatives in experienced centers.

Accumulating evidence have suggested that a subgroup of patients with severe COVID-19 may have a cytokine storm syndrome. ${ }^{[3]}$ We recommend identification and treatment of hyperinflammation using existing, approved therapies with proven safety profiles to address the immediate need to reduce the rising mortality rates. Secondary hemophagocytic lymphohistiocytosis is a syndrome characterized by a fulminant and fatal hypercytokinemia associated with multiorgan failure. All patients should be screened for hyperinflammation using laboratory trends (e.g., elevated ferritin, decreased platelet counts, or erythrocyte sedimentation rate) to identify the

Received: April 06, 2020 Accepted: April 07, 2020 Published online: April 22, 2020

Correspondence: Serdar Günaydın, MD, PhD. Ankara Şehir Hastanesi Kalp ve Damar Cerrahisi Kliniği, 06800 Çankaya, Ankara, Türkiye.

Tel: +90 536 - 3896521 e-mail: sgunaydin@isnet.net.tr 
subgroup of patients in whom immunosuppression can improve mortality. Therapeutic options include steroids, intravenous immunoglobulin, selective cytokine blockade (e.g., anakinra or tocilizumab) and janus kinase inhibition. Cytokine filtration (extracorporeal blood purification) is approved by the United States Food and Drug Administration (FDA) to treat patients 18 years of age or older with confirmed COVID-19 admitted to the operating room/intensive care unit with confirmed or imminent respiratory failure to reduce pro-inflammatory cytokines levels. ${ }^{[3]}$

The issue of potential risk for aerosolization/ contamination of virus via oxygenator/chest drains is also an under-recognized means of viral spread, which may put patients and healthcare professionals at an utmost risk for infection. Although most membrane oxygenators used today are surface coated, there is no evidence-based in the literature to suggest that viruses cannot permeate these hollow fiber materials. The viruses in the Coronaviridae family (i.e., SARS) range in size between 0.08 and 0.15 microns. There is a direct blood to gas contact during $\mathrm{CPB}$, when the micropores are eventually coated with the patient's blood plasma proteins, after which gas exchange takes place through the micropores via direct contact. Over time, a decrease in the membrane's permeance due to the increase in plasma wetting can degrade an oxygenator's performance, which is one of the contributing factors to the international standard organization limiting all hollow fiber membrane oxygenator to use $<6 \mathrm{~h}$. It is possible to retrofit any filter to an outlet using reducing/increasing connectors and different tubing sizes. However, in the long-term use, this filter may lead to increasing resistance and pressure build-up inside the hollow fibers, which is a dangerous situation for sudden gas embolism. The filters do remove some viruses, although even in a ventilator circuit they get wet, increase resistance to flow and, therefore, have to be changed on a daily basis. Another option is to scavenge the membrane's gas exhaust port to the atmosphere using the hospital vacuum, as in the operating room. Just make sure to cut notch or place a connector in vacuum tubing to prevent membrane pressure build-up. There should be clear protocols and guidelines for handling of medical wastes from these specific patients..$^{[4,5]}$

Regarding the volume of aerosolization from a chest drain bottle, it is recommended to use of closed drainage systems, i.e., connecting the standard drain bottle to wall suction to avoid the spread of viral load via aerosolization. However, to achieve this goal, the safety valve must be occluded with a potential risk for increasing intrathoracic pressure and causing tension, and should the suction system be switched off, whilst still connected to the bottle. Furthermore, keeping the bottle attached to wall suction significantly limits the mobilization of patients, which is a significant risk factor for postoperative complications in the surgical patient. To overcome this issue, a possible consideration would be to attach an antimicrobial filter, such as those used in the ventilator circuits, to the chest drain suction port leaving the drain off suction and occluding the safety valve. Connecting the filter directly to the chest drain should be discouraged, as fluid and moisture directly from the chest cavity are likely to interfere with the functioning of the filter.

Across the world, the medical care is hampered by a critical shortage of not only equipment, but also impediments to the blood supply. Although it does not appear very likely that the virus can be transmitted through allogeneic blood transfusion, it still remains to be fully elucidated. Patient blood management should be considered as a strategic approach in times, when there is an urgent need to optimize healthcare resources and reduce the pressure on the blood supply. Transfusion therapies can be often avoided by the utilization of clotting factors such as prothrombin complex concentrate or fibrinogen concentrate. In addition to transfusion-sparing effects, clotting factors also decrease the risk for transfusion-related complications, such as transfusion-related acute lung injury and transfusion-associated circulatory overload, the leading causes of morbidity and mortality. Antifibrinolytic agents, including tranexamic acid and epsilon aminocaproic acid, are widely acceptable, inexpensive and highly effective safe pharmacologic agents which can be utilized to stabilize clot formation and prevent hyperfibrinolysis. ${ }^{[6]}$ Cell salvage, which involves the collection of the patient's own blood loss, filtering and washing to ensure the removal of impurities, and direct return of the autologous component to the patient, is associated with reduced utilization of the allogeneic blood component. Therefore, cell salvage is recommended for all procedures with CPB and/or ECMO/ECLS ${ }^{[7]}$

In conclusion, the world is united regarding the goal of ending the COVID-19 pandemic. Advice given at the beginning of this journey should be updated as we learn more. This disease has distinct phases and treatment will differ as patients move through. ${ }^{[8,9]}$

\section{Declaration of conflicting interests}

The author declared no conflicts of interest with respect to the authorship and/or publication of this article. 


\section{Funding}

The author received no financial support for the research and/or authorship of this article.

\section{REFERENCES}

1. Mavioğlu HL, Ünal EU, Aşkın G, Küçüker ŞA, Özatik MA. Perioperative planning for cardiovascular operations in the COVID-19 pandemic. Turk Gogus Kalp Dama 2020;28:236-43.

2. Tang YW, Schmitz JE, Persing DH, Stratton CW. The Laboratory Diagnosis of COVID-19 Infection: Current Issues and Challenges. J Clin Microbiol 2020. pii: JCM.00512-20. [Epub ahead of print]

3. Gunaydin S, McCusker K, Vijay V. Strategic leukofiltration in cardiac surgery. Curr Med Chem Cardiovasc Hematol Agents 2005;3:323-31.

4. Gunaydin S. Clinical significance of coated extracorporeal circuits: a review of novel technologies. Perfusion 2004;19 Suppl 1:S33-41.

5. Gunaydin S. Emerging technologies in biocompatible surface modifying additives: quest for physiologic cardiopulmonary bypass. Curr Med Chem Cardiovasc Hematol Agents 2004;2:295-302.

6. Gunaydin S, McCusker K. The evolution of patient blood management programs in cardiac surgery: what is the ultimate frontier? Presented at the 57th AmSECT International Conference; 2019 Mar 8-10; Nashville, TN, USA.

7. Gunaydin S, Gourlay T. Novel ultrafiltration technique for blood conservation in cardiac operations. Ann Thorac Surg 2013;95:2148-51.

8. Angus DC. Optimizing the Trade-off Between Learning and Doing in a Pandemic. JAMA 2020. [Epub ahead of print]

9. Demirkılıç U, Gunaydin S, Doğancı S, Ural Z, editors. Extracorporeal Membrane Oxygenation (ECMO.) Ankara: Turkish Clinics Publishing; 2014. 